Article

\title{
Organo-Selenium-Containing Polyester Bandage Inhibits Bacterial Biofilm Growth on the Bandage and in the Wound
}

\author{
Phat Tran ${ }^{1}$, Tyler Enos ${ }^{1}$, Keaton Luth ${ }^{1}$, Abdul Hamood ${ }^{2}$, Coby Ray ${ }^{1}$, Kelly Mitchell ${ }^{1}$ and \\ Ted W. Reid 1,3,*(D) \\ 1 Department of Ophthalmology \& Visual Sciences, TTUHSC, Lubbock, TX 79430, USA; \\ Phat.tran@ttuhsc.edu (P.T.); tyler.enos@phhs.org (T.E.); keaton.luth@gmail.com (K.L.); \\ Coby.ray@ttuhsc.edu (C.R.); Kelly.mitchell@ttuhsc.edu (K.M.) \\ 2 Department of Microbiology \& Immunology, TTUHSC, Lubbock, TX 79430, USA; abdul.hamood@ttuhsc.edu \\ 3 SelenBio, Inc., Austin, TX 78735, USA \\ * Correspondence: ted.reid@TTUHSC.edu; Tel.: +1-806-743-9982
}

Received: 22 February 2020; Accepted: 12 March 2020; Published: 17 March 2020

\begin{abstract}
The dressing material of a wound plays a key role since bacteria can live in the bandage and keep re-infecting the wound, thus a bandage is needed that blocks biofilm in the bandage. Using an in vivo wound biofilm model, we examined the effectiveness of an organo-selenium (OS)-coated polyester dressing to inhibit the growth of bacteria in a wound. Staphylococcus aureus (as well as MRSA, Methicillin resistant Staph aureus), Stenotrophomonas maltophilia, Enterococcus faecalis, Staphylococcus epidermidis, and Pseudomonas aeruginosa were chosen for the wound infection study. All the bacteria were enumerated in the wound dressing and in the wound tissue under the dressing. Using colony-forming unit (CFU) assays, over 7 logs of inhibition (100\%) was found for all the bacterial strains on the material of the OS-coated wound dressing and in the tissue under that dressing. Confocal laser scanning microscopy along with IVIS spectrum in vivo imaging confirmed the CFU results. Thus, the dressing acts as a reservoir for a biofilm, which causes wound infection. The same results were obtained after soaking the dressing in PBS at $37^{\circ} \mathrm{C}$ for three months before use. These results suggest that an OS coating on polyester dressing is both effective and durable in blocking wound infection.
\end{abstract}

Keywords: anti-biofilm formation; wound healing; organo-selenium; dressing technology

\section{Introduction}

Organo-selenium, unlike other biocidal agents, such as silver ions, can be covalently attached to various materials with no loss of catalytic activity. Previous studies have shown that selenium (Se) covalently bound to a solid matrix retains its ability to catalyze the formation of superoxide radicals $\left(\mathrm{O}_{2}{ }^{-}\right)$in a continuous process in which Se donates an electron to oxygen and acquires one from sulfur compounds (such as glutathione) that are present in all body fluids [1]. Recently, it has been shown that organo-selenium can be attached to different biomaterials and medical devices to block the formation of P. aeruginosa and S. aureus biofilms on those materials [2-6]. Once a biofilm is formed on one of these devices, the only remedy is to replace the infected device, making prevention paramount. Selenium has additionally shown promise for this purpose in that unlike other antibacterial agents, longer periods of exposure to an aqueous environment do not compromise the effectiveness of the coating [2-6].

Biofilm formation occurring in a wound itself inhibits wound healing [7,8]. Antibiotics or silver embedded in gauze have been shown to be efficacious in preventing wound infection [9]. However, there are several problems associated with these treatments. One is the increasing number 
of antibiotic-resistant bacteria, including P. aeruginosa and S. aureus, which severely limits the choices for antibiotic treatment. A second is that in order to be effective, silver compounds must leach into the surrounding tissue, which can cause tissue damage [9]. Silver also discolors the wound, which makes it hard to assess wound healing. Because of these problems it is essential to investigate alternative antibacterial agents that can contact the infected tissues and prevent the development of bacterial biofilms. In this study, we examined the effectiveness of a selenium-coated polyester for inhibiting the growth of different bacteria. Polyester is used in medicine primarily as a bandage material. The surface is susceptible to biofilm formation, which can cause an infection of the wound. The idea of this study is not only to prevent biofilm formation in the bandage but to lessen the chance of biofilm formation in the wound.

\section{Experimental Section}

\subsection{Coating Polyester Dressing with Polymerized Se-AAEMA}

The Se-AAEMA [seleno-2 (methacryloyloxy)ethyl acetoacetate] monomer was purchased from Eburon Organics International (catalog No. 700.010; Lubbock, TX, USA). Se-AAEMA (22\% [wt/wt] selenium) stock solution was dissolved and diluted in 99.99\% 2-(methacryloyloxy)-ethyl acetoacetate (AAEMA) to yield the 1\% Se-AAEMA (wt/wt), used for the coating. Then, $100 \%$ ivory polyester fabric purchased from a local Walmart store (\#M30-23) was cut into 4 inch $x 4$ inch square pieces and soaked in sterilized distilled water overnight. The washed polyester dressing was then dried at $37^{\circ} \mathrm{C}$ in an oven and coated with AAEMA or Se-AAEMA. Briefly, the dressings were soaked in AAEMA or Se-AAEMA for $30 \mathrm{~min}$. Excess AAEMA or Se-AAEMA was removed from the dressings by passing the soaked bandage through a roller press (mechanical cloth wringer). The AAEMA or Se-AAEMA polyester dressings were then sprayed with $\mathrm{H}_{2} \mathrm{O}_{2}$ using a bottle sprayer to initiate free radical polymerization. This has little or no effect on the bandage material. As a result of the in situ polymerization, the polymer is held in place by a combination of van der Waals forces and physical interlinking with the dressing material. The dressings were then transferred to a $66^{\circ} \mathrm{C}$ curing oven. After curing, the dressings were washed twice ( $1 \mathrm{~h}$ each) in sterile phosphate-buffered saline (PBS, $\mathrm{pH} \mathrm{7.4)}$ at $37^{\circ} \mathrm{C}$. The dressings were then dried, cut into $1 \mathrm{~cm} \times 1 \mathrm{~cm}$ and $1.5 \mathrm{~cm} \times 1.5 \mathrm{~cm}$ pieces, sterilized by dried autoclaving, dried again, and stored at room temperature until being used in the assays. All percentages listed for the different concentrations of coating are the percentages of selenium in the coating.

\subsection{Bacterial Strains}

All strains were grown in Luria Bertani (LB) Broth, Mueller Hinton Broth, or on LB Agar plates at $37^{\circ} \mathrm{C}$. S. aureus AH133 (TTUHSC laboratory strain) was used. This is a lab strain that constitutively expresses green fluorescent protein from a plasmid (pCM11) in the presence of $1 \mu \mathrm{g} / \mathrm{mL}$ erythromycin [10]. Pseudomonas aeruginosa strain PAO1/pMRP9-1was also grown. This strain carries the plasmid pMRP9-1 that contains the gene that codes for the green fluorescent protein [11]. To maintain the plasmid, the strain was grown in the presence of $300 \mu \mathrm{g} / \mathrm{mL}$ carbenicillin. The Methicillin-resistant S. aureus and S. epidermidis clinical isolates were obtained from leg wounds or abscess wounds from the Clinical lab at Texas Tech University Health Sciences Center under an approved Institutional Review Board protocol of the Texas Tech University Medical Center in Lubbock, Texas. Staphylococcus aureus Lux Xen29 was also used, a strain with a plasmid containing a gene that codes for the luciferase protein [12]. To maintain the plasmid, the strain was grown in the presence of $40 \mu \mathrm{g} / \mathrm{mL}$ kanamycin. This strain was available at our lab at Texas Tech University Health Sciences Center. Pseudomonas aeruginosa PAO1 Lux Xen5 strain was available at our lab at Texas Tech University Health Sciences Center [12]. Finally, Stenotrophomonas maltophilia ATCC ${ }^{\circledR} 53199^{\mathrm{TM}}$ was available at our lab at Texas Tech University Health Sciences Center. All bacterial stock was stored at $-80^{\circ} \mathrm{C}$. 


\subsection{Colony-Forming Unit (CFU) Assay In Vitro}

Bacteria were grown overnight, and washed once with PBS (pH 7.4). They were then re-suspended in PBS (pH 7.4) to an optical density $\left(\mathrm{OD}_{600}\right)$ of $0.1\left(10^{7} \mathrm{CFU} / \mathrm{mL}\right)$, and serially diluted 10-fold. All PBS assay solutions contained $150 \mu \mathrm{M}$ reduced glutathione (Sigma Chemical, St. Louis, MO, USA). Ten microliter aliquots containing $10^{2}$ to $10^{3}$ colony-forming units were added to the bandages. They were a stack (3 pieces of one square centimeter) containing either AAEMA (control treated with AAEMA with no selenium-listed in the figures as untreated dressing) polyester dressing, or Se-AAEMA test polyester dressing. After the bacterial inoculum was absorbed into the dressings, they were placed on LB agar plates, and the plates were incubated at $37^{\circ} \mathrm{C}$ for $24 \mathrm{~h}$. Biofilms were then quantified by determining the $\mathrm{CFU}$ per square centimeter of dressing by the following procedure. After incubation, each stack of dressing was rinsed with sterile PBS and then was transferred to a sterile $1.5-\mathrm{mL}$ microcentrifuge tube containing $1 \mathrm{~mL}$ of PBS $(\mathrm{pH}=7.4)$ for enumeration of bacteria. The tubes were placed in a water bath sonicator for 5-10 min to loosen the cells within the dressing and then vigorously vortexed 3 times for $1 \mathrm{~min}$ to detach the cells. Suspended cells were serially diluted (10-fold) in PBS, and $10-\mu \mathrm{L}$ aliquots of each dilution were spotted onto LB Agar plates. In addition, the remaining $900-\mu \mathrm{L}$ zero dilution sample was plated on a different LB Agar plate. Thus, the equation for back calculating the bacterial concentration was CFU $\times$ dilution factor $\times 100$, with the exception of the $900-\mu \mathrm{L}$ sample, which was calculated as CFU $\times 1$. This means that if only one bacterium was originally in the tube, we would have a $90 \%$ chance of detecting it. All experiments were done in triplicate, and all measurements were repeated three times.

\subsection{Mouse Wound Infection Model}

Six adult female Balb C mice (including a control group) weighing 20-24 g were anesthetized using a mixture of isoflurane and oxygen, and their backs were shaved. Shaved areas were completely cleansed with $95 \%$ ethanol. A flap of skin was lifted and a $1.0-\mathrm{cm}^{2}$ piece of skin was removed centrally in the shaved area with sterile scissors. Either an AAEMA control or Se-AAEMA dressing $(1.5 \mathrm{~cm} \times 1.5 \mathrm{~cm})$ was placed on the wound. The dressings were secured in place by applying a clear OPSITE dressing over the back of the mouse. Aliquots containing $10^{2}$ to $10^{3} \mathrm{CFU}$ of bacteria in $20 \mu \mathrm{L}$ were injected in the area between the bandage and the wound. The mice were monitored twice a day for signs of infection or distress. The dressings were removed, and the connective tissue around the wound and the tissue under the wound (down to the spinal cord) was then dissected and removed. The extracted dressings and all the dissected tissue were gently rinsed in PBS and homogenized in PBS. Excess PBS was drained from the dressing by touching it to a sterile filter paper and the dressing was then transferred to a sterile homogenized tube containing $2 \mathrm{~mL}$ of PBS for enumeration of bacteria by either determination of CFU or by confocal microscopy. All experiments were done at least in triplicate.

Tissue: At the end of the experiment (5 days), the mouse was euthanized (with $\mathrm{CO}_{2}$ in a closed chamber) and the connective tissue around the wound and the tissue under the wound (down to the spinal cord) was then dissected and removed. This was then homogenized and used for a CFU assay in a manner similar to that used for the dressing above.

Animals were treated in accordance with the protocol approved by the Institutional Animal Care and Use Committee at Texas Tech University Health Sciences Center in Lubbock, TX, USA.

\subsection{Biofilm Detection by Assay Using the Dressing}

Biofilms were quantified by determining the CFU recovered from the dressings. As above, after removal from the mouse, each piece of dressing was gently washed twice with sterile $1 \times$ PBS to remove any planktonic bacteria. Excess PBS was drained from the dressing by using sterile filter paper and the dressing was then transferred to a sterile homogenized tube containing $2 \mathrm{~mL}$ of sterile $1 \times \mathrm{PBS}$ (pH 7.4). The dressings were homogenized to loosen the cells within the biofilm and then vigorously vortexed 3 times for $1 \mathrm{~min}$ to detach the cells. Suspended cells were serially diluted (10-fold) in sterile $1 \times$ PBS 
( $\mathrm{pH}$ 7.4), and 10- $\mathrm{LL}$ aliquots of each dilution were spotted onto LB Agar plates. Bacterial counts were determined as described above. The results were reported as attached CFU per dressings recovered from each mouse. All experiments were done in triplicate.

\subsection{Biofilm Detection Assay for Tissue}

At the end of the in vivo experiment (5 days), the mice were euthanized, and the tissue of the wounds was removed. The tissue was weighed, and it was then homogenized. The CFU assay was performed in a manner similar to the assay used for the dressing above. The results were reported as attached CFU per gram of tissue. All experiments were done in at least triplicate.

\subsection{Biofilm Analysis by Fluorescence Microscopy}

The pieces of polyester dressings at the end of the in vivo 5-day incubation were assayed as described above for the CFU assays. AAEMA control and Se-AAEMA polyester dressing segments from three mice of each group were also examined for the presence of biofilm by confocal laser scanning microscopy (CLSM) using an Olympus Fluoview FV300 (Olympus America, Center Valley, PA, USA). Animals were treated in accordance with the protocol approved by the Institutional Animal Care and Use Committee at Texas Tech University Health Sciences Center in Lubbock, TX, USA.

\subsection{In Vivo Live Imaging Studies}

Mice were also infected with either Staphylococcus aureus Lux Xen29 or Pseudomonas aeruginosa Lux Xen 5 and observed at day 5 after treatment. The mice were lightly anesthetized, and the infected wounds were visualized using an IVIS Lumina XR system with Living Image software (Perkin Elmer, US). This instrument allowed us to directly detect pathogenic infections in living animals by producing images through bioluminescence. After imaging, the mice were euthanized, and the gauze dressing and wound tissues were recovered. The samples were then analyzed by the CFU assay as described above. Imaging experiments were conducted at the Texas Tech University Health Sciences Center Image Analysis Core Facility (Lubbock, TX, USA). All experiments were done in triplicate.

\subsection{Analysis of the Long-Term Stability of the Organo-Selenium-Containing Polyester Dressing}

One square-centimeter strips of the organo-selenium-containing polyester dressing were completely immersed in $1.0 \mathrm{~mL}$ of PBS in sterile glass tubes and incubated at $37^{\circ} \mathrm{C}$ for a month. At the end of the incubation period, the pieces were removed, dried, sterilized by dried autoclaving, and utilized in the in vitro biofilm assay, as described above, for determination of the durability of the anti-biofilm activity of the coating.

\subsection{Statistical Analysis}

Results of the CFU assays were analyzed with Prism ${ }^{\circledR}$ version 4.03 (GraphPad Software, San Diego, CA, USA) with $95 \%$ confidence intervals (CIs) of the difference. Comparisons of the in vivo biofilms formed on Se-free and Se-AAEMA polyester dressings were analyzed by a two-tailed unpaired $\mathrm{t}$-test to determine significant differences. All experiments were done at least in triplicate.

\section{Results}

\subsection{In Vitro Testing of the Selenium-Coated Polyester Bandage against Biofim Formation by Different Bacterial Strains}

For the bandages coated with organo-selenium in the form of 1\% Se-AAEMA (see Figure 1), after $24 \mathrm{~h}$ of growth, between 7-8 logs of inhibition were seen (by CFU assay) for all the different bacterial strains (Stenotrophomonas maltophilia, Enterococcus faecalis, Staphylococcus epidermidis and Pseudomonas aeruginosa), when compared with the control bandage coated with AAEMA containing no selenium. This result was true for all bacteria, whether Gram-negative or Gram-positive. 
A Staphylococcus aureus GFP AH133

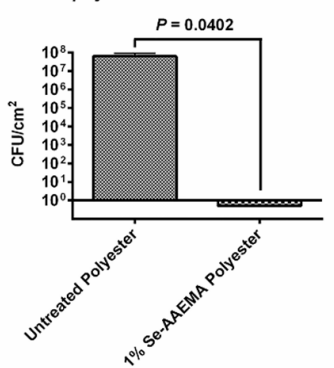

D Enterococcus faecalis GFP

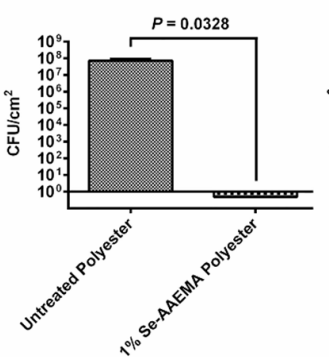

B

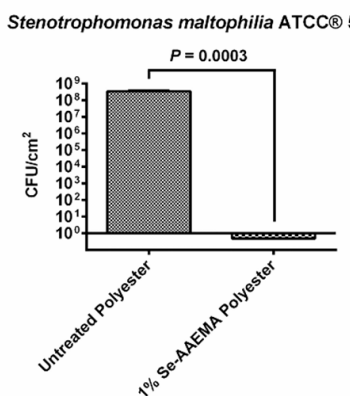

E $\begin{gathered}\text { Methicillin-resistant } \\ \text { Staphylococcus aureus } \mathrm{Cl} 1\end{gathered}$

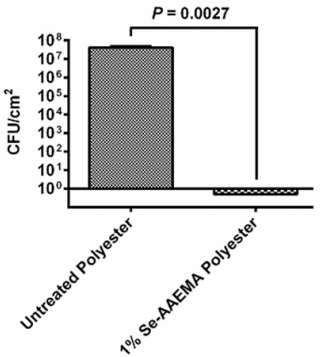

$\mathbf{F}$

Methicillin-resistant Staphylococcus aureus $\mathrm{Cl} 2$

C

Pseudomonas aeruginosa PAO1 GFP

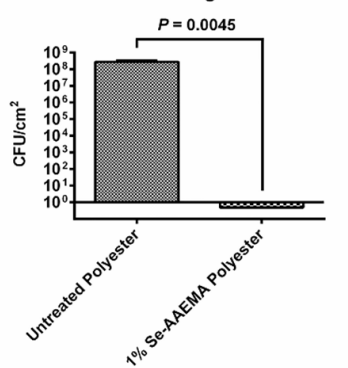

G

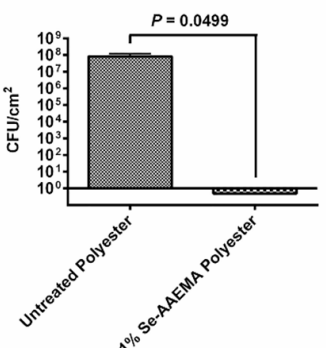

Figure 1. Graph of the colony-forming units of (A) Staphylococcus aureus GFP AH133, (B) Stenotrophomonas maltophilia ATCC ${ }^{\circledR}$ 53199 Methicillin-resistant Staphylococcus aureus CI 1, (F) Methicillin-resistant Staphylococcus aureus CI 2, and (G) Staphylococcus epidermidis CI biofilms formed on untreated polyester and 1\% Se-AAEMA polyester. Values represent the means of triplicate experiments \pm SEM. A two-tailed unpaired $t$ test was used to determine statistical significance. Untreated polyester has AAEMA but no selenium.

3.2. In Vitro Visualization of Biofilm Formation by Confocal Laser Scanning Microscopy for Different Bacteria on a Polyester Bandage with and without a Selenium Coating

As can be seen in Figure 2, biofilms were formed for $24 \mathrm{~h}$ on the polyester that was only coated with AAEMA, while no biofilm was formed on the polyester with the selenium-AAEMA. The non-selenium-coated fibers were seen to serve as a good binding material for the different bacteria. These results are consistent with those found from the CFU studies seen in Figure 1.

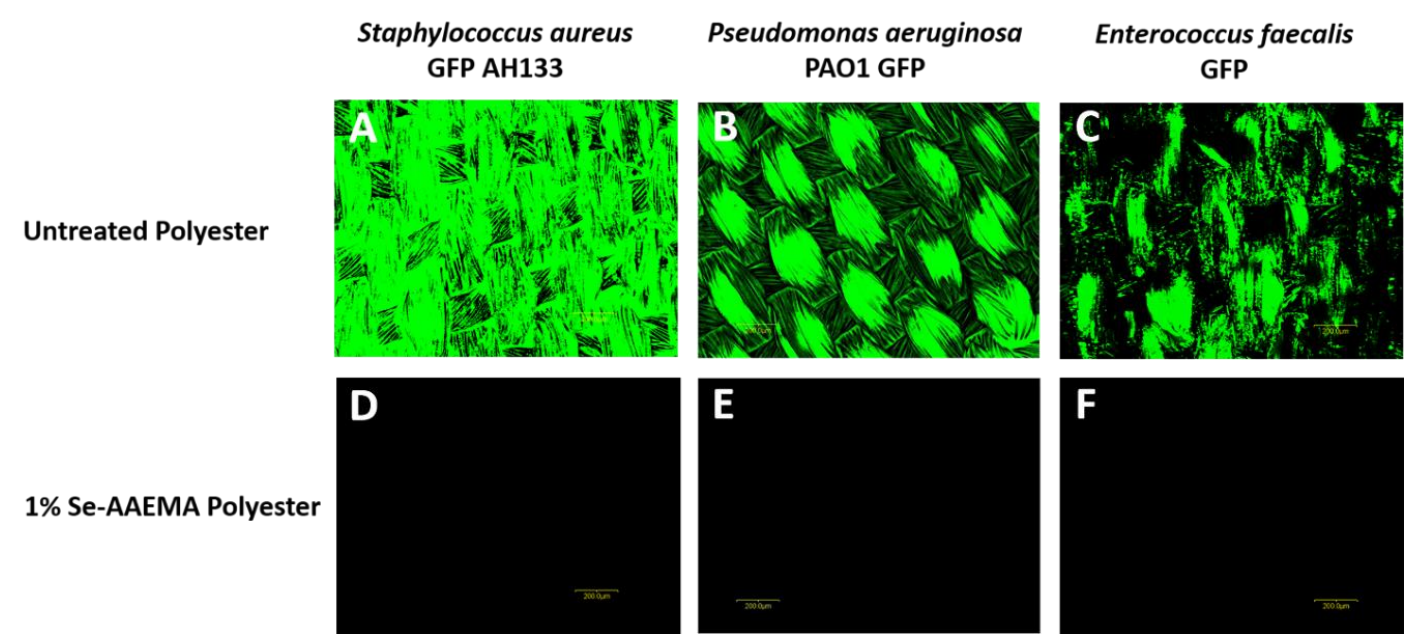

Figure 2. In Vitro study. Representative confocal laser scanning microscopy images of (A) Staphylococcus aureus GFP AH133, (B) Pseudomonas aeruginosa PAO1 GFP, and (C) Enterococcus faecalis GFP biofilm formed on untreated polyester and 1\% Se-AAEMA polyester. Untreated polyester has AAEMA but no selenium. (D-F) are the same samples as those above however they have Se-AAEMA. As seen all the bacteria are eliminated. The bar is $200 \mu \mathrm{m}$. 
3.3. In Vivo Studies of Biofilm Formation on a Polyester Dressing and in the Underlying Wound on a Mouse Wound Model

Different bacteria were injected under a polyester bandage dressing, either with or without a selenium coating, which covered a mouse wound model. These were then allowed to grow for 5 days. As shown in Figure 3, not only did the selenium coating result in between a 7-8 logs reduction (100\%) in the amount of biofilm on the dressing compared with the non-selenium containing dressing, but it also produced a $7-8 \operatorname{logs}$ of reduction $(100 \%)$ in the underlying tissue of the wound. These results were found for Staphylococcus aureus, Pseudomonas aeruginosa, and two different clinical strains of MRSA. These results are confirmed by confocal laser scanning microscopy in Figure 4.
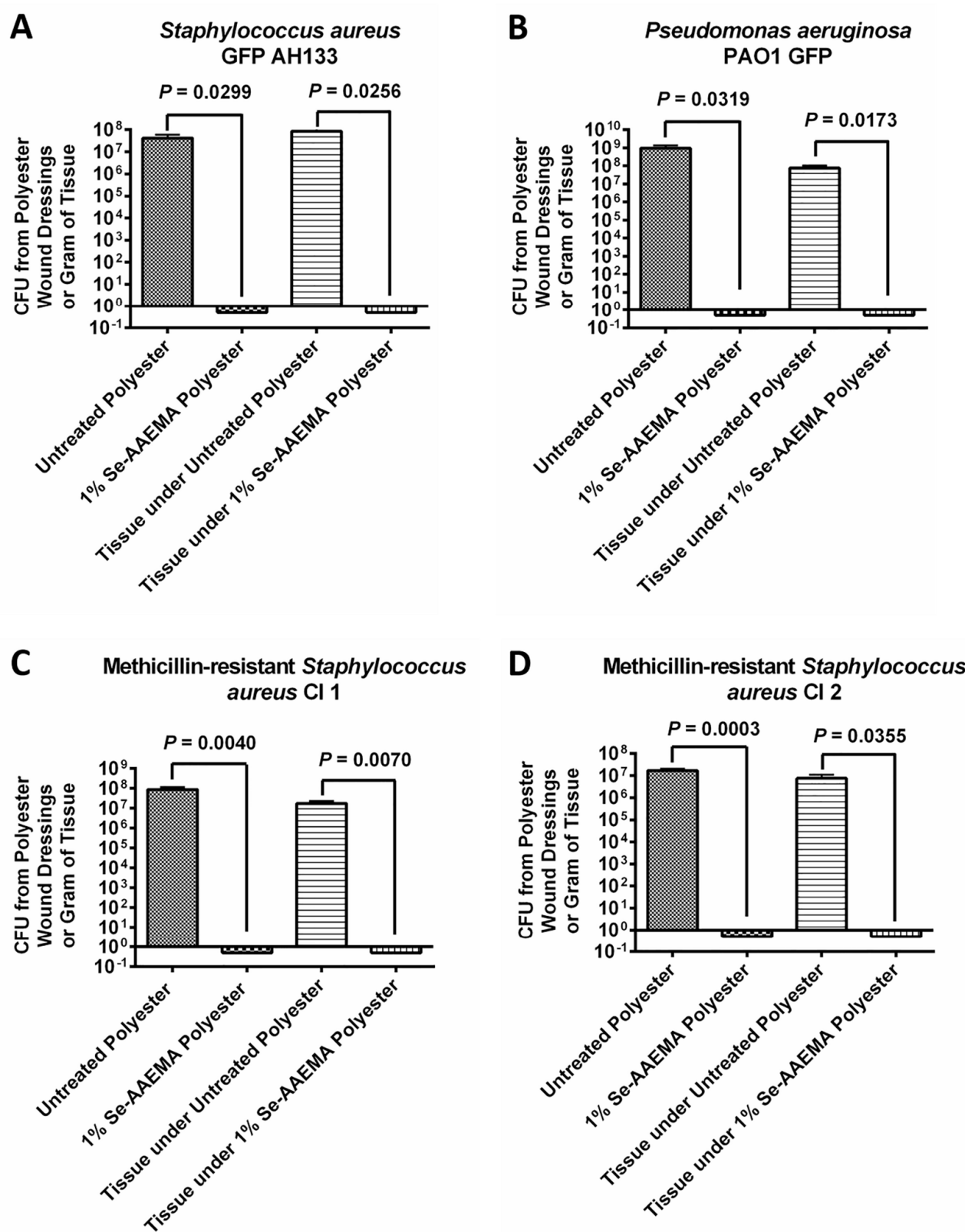

Figure 3. Graph of the colony-forming units of (A) Staphylococcus aureus GFP AH133, (B) Pseudomonas aeruginosa PAO1 GFP, (C) Methicillin-resistant Staphylococcus aureus CI 1, and (D) Methicillin-resistant Staphylococcus aureus CI 2 biofilms formed on the polyester dressings and in the tissue under the polyester dressings on a mouse wound. Values represent the means of six replicate experiments \pm SEM. A two-tailed unpaired $t$ test was used to determine statistical significance. Untreated polyester has AAEMA but no selenium. 


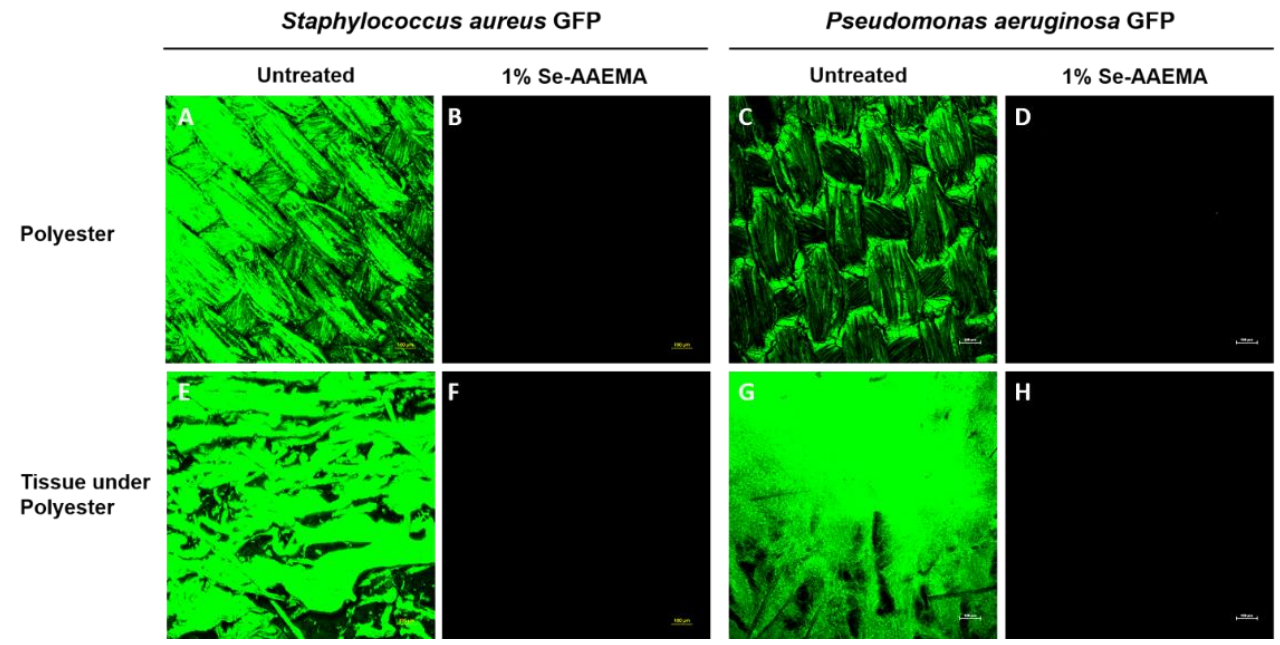

Figure 4. Mouse wounds after 5 days. Representative confocal laser scanning microscopy images of (A) Staphylococcus aureus GFP AH133 with untreated polyester showing bacteria in the AAEMA bandage, and $(\mathbf{B})$ is the same except it is with a Se-AAEMA bandage, while (E) is the tissue under bandage (A), and (F) is the tissue under bandage (B). (C) is Pseudomonas aeruginosa PAO1 GFP biofilms formed on the AAEMA treated polyester dressings and (D) is the same as (A) except it is with the SeAAEMA treated dressing. (G) is the tissue under the polyester dressings $(\mathbf{A})$, and $(\mathbf{H})$ is the tissue under the SeAAEMA bandage (D). Bar is $100 \mu \mathrm{m}$.

\subsection{In Vivo Live Imaging of the Growth of Bacteria in the Wound}

In order to image the growth of bacteria in the wound, bacteria with the Lux gene were used. Mice were infected with either Staphylococcus aureus Lux Xen29 or Pseudomonas aeruginosa Lux Xen5 under the polyester bandage and observed at day 5 after treatment. The actual growth of bacteria with this gene present is seen in Figure 5 for bacteria in the dressing and in the wound. As shown in the pictures (Figure 6), no active infection could be detected in under the Se-AAEMA polyester dressing while each mouse with a control (AAEMA) bandage demonstrated obvious active wound infection.

\section{A}

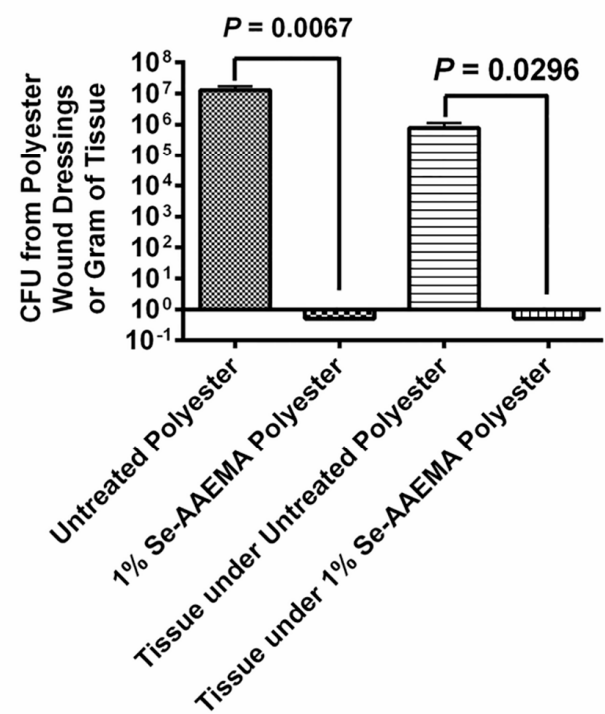

B

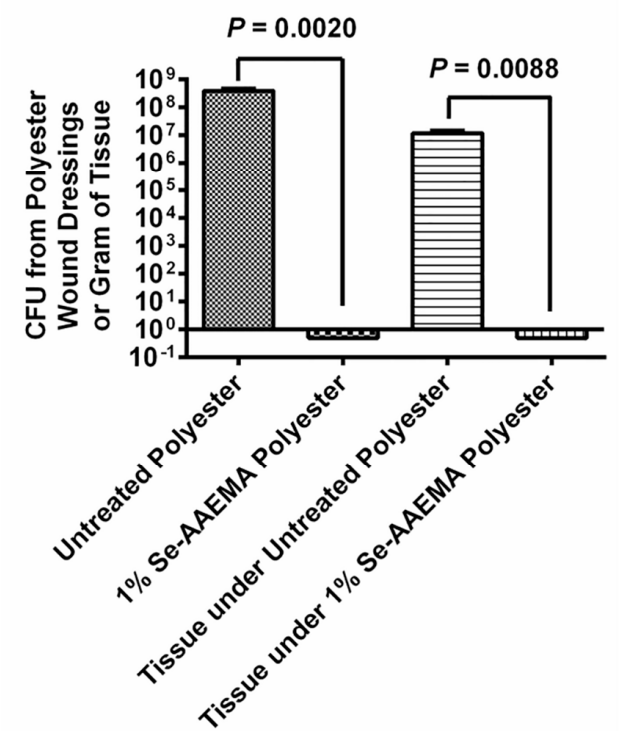

Figure 5. Graphs of the colony-forming units recovered from the effect of AAEMA and SeAAEMA coated polyester dressing (A) S. aureus Lux Xen29 and (B) P. aeruginosa Lux Xen5 in the polyester dressings and the mouse wound tissue. Untreated polyester has AAEMA but no selenium. 


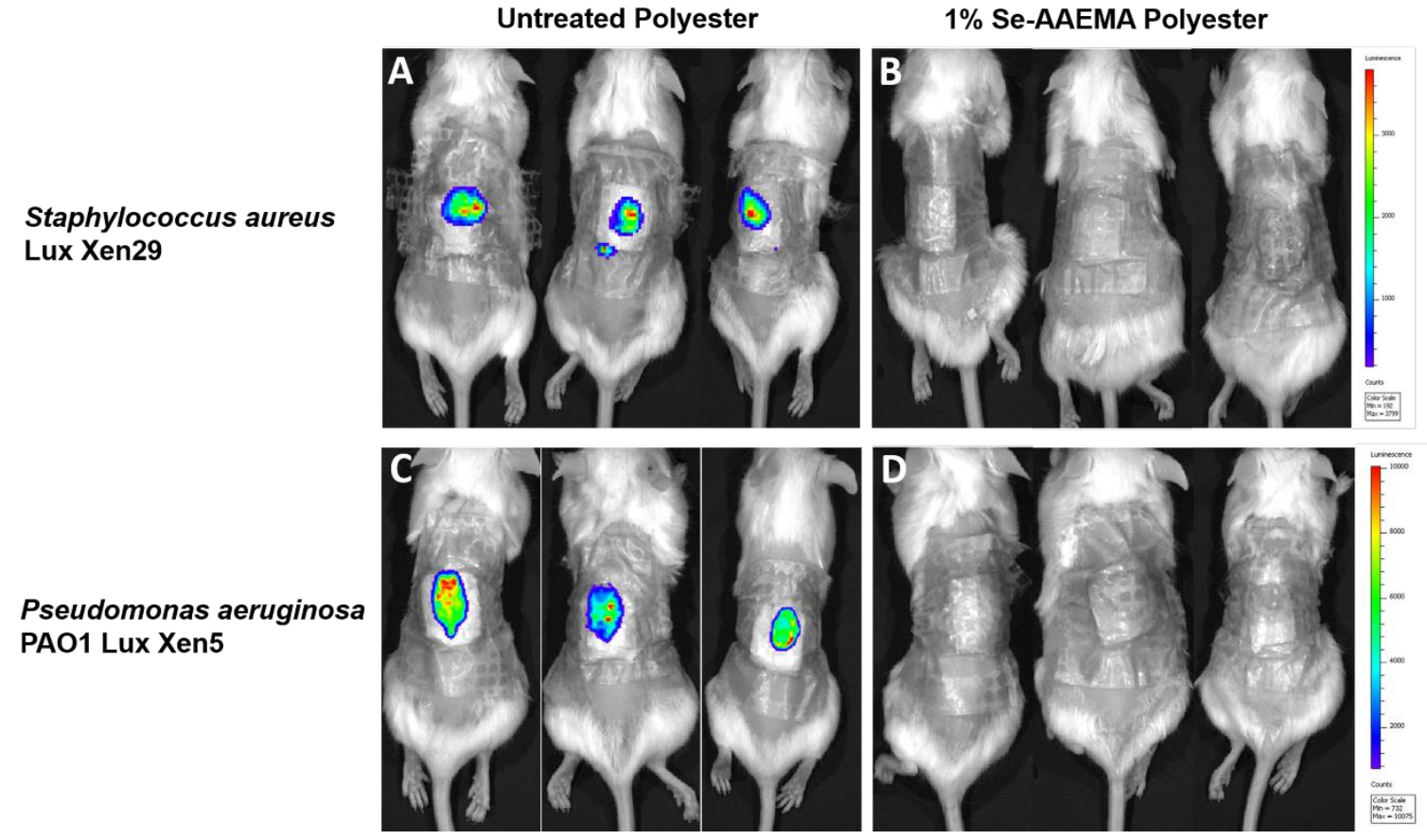

Figure 6. Representative IVIS in vivo live images of (A) S. aureus Lux Xen29 under AAEMA polyester dressing and (B) Se-AAEMA polyester dressing. (C) P. aeruginosa Lux Xen5 biofilms formed under the AAEMA polyester dressings and (D) the Se-AAEMA polyester dressing.

\subsection{Stability of the Selenium-Coated Polyester Bandage in Preventing Biofilm Formation}

A stability study was carried out to see if the organo-selenium-coated bandages retained their biofilm inhibitory activity after one month of storage in PBS solution at $37^{\circ} \mathrm{C}$. As seen in Figure 7 , there was still $8 \operatorname{logs}(100 \%)$ of inhibition present against S. aureus GFP AH133 with the 1\% Se-AAEMA coating. These results were confirmed by visualization with the confocal laser scanning microscope (Figure 7B,C).

A

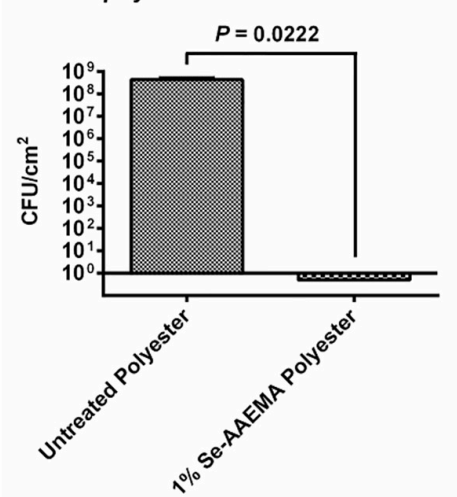

Staphylococcus aureus GFP AH133

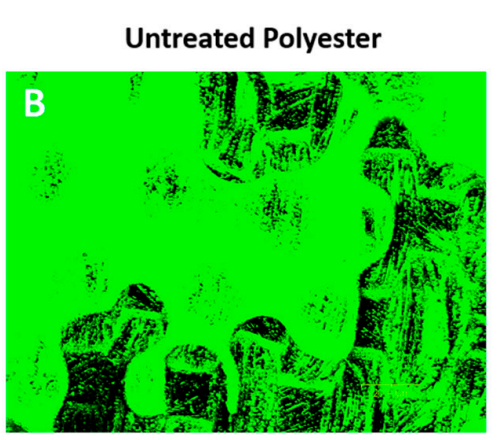

1\% Se-AAEMA Polyester

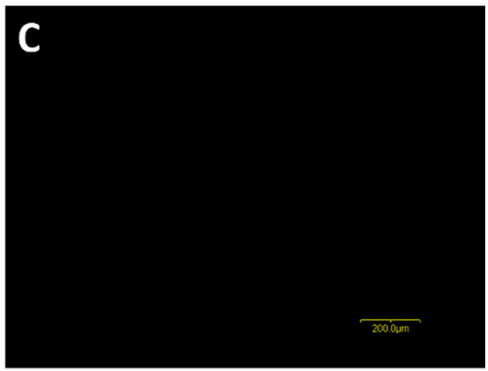

Figure 7. Stability study of bandage after one month in PBS at $37^{\circ} \mathrm{C}$. The inhibitory effect of the organo-selenium coating is long-lasting against Staphylococcus aureus GFP AH133 biofilms formed on untreated polyester and $1 \%$ Se-AAEMA polyester, which were previously soaked in $1 \times \mathrm{PBS}(\mathrm{pH}=7.4)$ for three months. Values represent the means of quadruplicate experiments \pm SEM. (A) two-tailed unpaired $t$ test was used to determine statistical significance. Representative confocal laser scanning microscopy images of (B) Staphylococcus aureus GFP AH133 biofilms formed on untreated polyester and (C) $1 \%$ Se-AAEMA polyester, which were previously soaked in $1 \times \mathrm{PBS}(\mathrm{pH}=7.4)$ for three months. Untreated polyester has AAEMA but no selenium. Bar is $200 \mathrm{~m}$. 


\section{Discussion}

These experiments studied whether selenium atoms that are covalently incorporated into a methacrylate polymer (AAEMA) used to coat a polyester bandage could block bacterial attachment to the bandage and influence biofilm formation in a wound. Selenium is unique amongst the elements in its ability to be attached to an organic molecule with a covalent bond and yet still retain its ability to catalyze the formation of superoxide radicals. This is done by selenium donating an electron to oxygen to form superoxide, resulting in the oxidation of the selenium atom. The oxidized selenium atom can then react with sulfhydryl groups present in all body fluids, such as glutathione, to become reduced, resulting in oxidization of the sulfhydryl (disulfide bond formation). This completes the catalytic cycle by essentially taking an electron from sulfur and giving it to oxygen. The advantage of this mechanism is that the selenium remains unchanged and attached to the polymer yet creates a local layer of superoxide on the surface of the material. However, the half-life of superoxide is short and does not travel far. It is probably due to this short half-life that previous studies have shown that there is no toxic effect upon nearby mammalian cells [2,4].

Based upon the mechanism described above, we expected the selenium coating to block bacterial attachment to the dressing. However, we were interested to find that the coating on the bandage also inhibited biofilm formation in the wound as well, even though the bacteria were injected into the area between the bandage and the wound. Since selenium does not leave the bandage and the half-life of the generated superoxide is very short (milliseconds at best), we expected the selenium mechanism to have more limited effects upon the wound tissue, especially during the 5 days of the experiment. One possible mechanism for the effect of the selenium coating on wound infection is that biofilm formation in a typical wound requires instigation by biofilm formation on the bandage. After the injection of the bacteria, the organisms that initially go into the wound are probably destroyed by the normal host cell defense mechanisms. Those bacteria that invade the control bandage, however, can form a biofilm on the bandage. This biofilm can release planktonic bacteria and toxins over time and continuously re-infect the wound. During this re-infection, toxins will kill cells in the wound, and these dead or compromised cells can serve as a matrix for biofilm formation in the wound itself. However, with the selenium-coated bandage, there is no biofilm formation in the bandage since the coating kills $100 \%$ of the bacteria that attempt to attach to the bandage. This serves to protect the wound from re-infection. Thus, a selenium-coated bandage should allow the wound requisite time to heal without biofilm formation. This was also confirmed by bioluminescence, which offers a method for monitoring infections in vivo over a period of several days. It is sensitive and non-invasive and requires fewer animals than conventional methodologies. It showed no infection developing in the selenium bandage or the wound over time.

\section{Conclusions}

Organo-selenium monomers can be polymerized into a coating on polyester fabric, which is suitable for medical wound dressings. Organo-selenium-coated polyester dressings significantly inhibited biofilm formation by all bacteria tested (both Gram-negative and Gram-positive). Of importance was the fact that the selenium coating not only inhibited biofilm formation in the dressing but also inhibited biofilm formation in an inoculated wound over a 5-day period. The organo-selenium coating on the polyester dressing was also found to be stable after long soaking, and the inhibitory effect on bacterial biofilm remained. In future experiments, we plan to test the incorporation of the organo-selenium monomer into the polyester polymer itself rather than just coating it on the surface of the dressing. This would likely make a superior dressing that avoids problems with surface wear, leaching, or abrasion, and would allow for more flexibility in manufacturing.

Author Contributions: T.W.R. and P.T. conceived and designed the experiments; P.T., T.E., K.L. performed the experiments; T.W.R., P.T., A.H., C.R. and K.M. analyzed the data; T.W.R. and P.T. wrote the paper. All authors have read and agreed to the published version of the manuscript. 
Funding: This research was supported by the South Plains Foundation Grant, Lubbock, Texas, USA, and SelenBio, Inc., Austin, Texas, USA.

Conflicts of Interest: The authors declare no conflict of interest, except T.W.R." T.W.R has a financial interest in SelenBio.

\section{References}

1. Chaudiere, J.; Courtin, O.; Leclaire, J. Glutathione oxidase activity of selenocystamine: A mechanistic study. Arch. Biochem. Biophys. 1992, 296, 328-336. [CrossRef]

2. Mathews, S.M.; Spallholz, J.E.; Grimson, M.J.; Dubielzig, R.R.; Gray, T.; Reid, T.W. Prevention of bacterial colonization of contact lenses with covalently attached selenium and effects on the rabbit cornea. Cornea 2006, 25, 806-814. [CrossRef] [PubMed]

3. Tran, P.L.; Hammond, A.A.; Mosley, T.; Cortez, J.; Gray, T.; Colmer-Hamood, J.A.; Shashtri, M.; Spallholz, J.E.; Hamood, A.N.; Reid, T.W. Organoselenium coating on cellulose inhibits the formation of biofilms by Pseudomonas aeruginosa and Staphylococcus aureus. Appl. Environ. Microbiol. 2009, 75, 3586-3592. [CrossRef] [PubMed]

4. Tran, P.L.; Lowry, N.; Campbell, T.; Reid, T.W.; Webster, D.R.; Tobin, E.; Aslani, A.; Mosley, T.; Dertien, J.; Colmer-Hamood, J.A.; et al. An organoselenium compound inhibits Staphylococcus aureus biofilms on hemodialysis catheters in vivo. Antimicrob. Agents Chemother. 2012, 56, 972-978. [CrossRef] [PubMed]

5. Tran, P.; Hamood, A.; Mosley, T.; Gray, T.; Jarvis, C.; Webster, D.; Amaechi, B.; Enos, T.; Reid, T. Organo-selenium-containing dental sealant inhibits bacterial biofilm. J. Dent. Res. 2013, 92, 461-466. [CrossRef] [PubMed]

6. Wang, J.C.; Tran, P.L.; Hanes, R.; Cordero, J.; Marchbanks, J.; Reid, T.W.; Colmer-Hamood, J.A.; Hamood, A.N. Inhibition of otopathogenic biofilms by organoselenium-coated tympanostomy tubes. JAMA Otolaryngol. Head Neck Surg. 2013, 139, 1009-1016. [CrossRef] [PubMed]

7. Hess, C.T. Clinical Guide: Skin and Wound Care, 6th ed.; Lippincott Williams \& Wilkins: Philadelphia, PA, USA, 2008; pp. 297-360.

8. Slachta, P.A. Caring for chronic wounds: A knowledge update. Am. Nurse Today 2008, 3, 27-32.

9. Wyllie, D.H.; Crook, D.W.; Peto, T.E. Mortality after Staphylococcus aureus bacteraemia in two hospitals in Oxfordshire, 1997-2003: Cohort study. BMJ (Clinical research ed.) 2006, 333, 281. [CrossRef] [PubMed]

10. Malone, C.L.; Boles, B.R.; Lauderdale, K.J.; Thoendel, M.; Kavanaugh, J.S.; Horswill, A.R. Fluorescent reporters for Staphylococcus aureus. J. Microbiol. Methods 2009, 77, 251-260. [CrossRef] [PubMed]

11. Holloway, B.W.; Krishnapillai, V.; Morgan, A.F. Chromosomal genetics of Pseudomonas. Microbiol. Rev. 1979, 43, 73-102. [CrossRef] [PubMed]

12. Miller, K.G.; Tran, P.L.; Haley, C.L.; Kruzek, C.; Colmer-Hamood, J.A.; Myntti, M.; Hamood, A.N. Next science wound gel technology, a novel agent that inhibits biofilm development by gram-positive and gram-negative wound pathogens. Antimicrob. Agents Chemother. 2014, 58, 3060-3072. [CrossRef] [PubMed]

(C) 2020 by the authors. Licensee MDPI, Basel, Switzerland. This article is an open access article distributed under the terms and conditions of the Creative Commons Attribution (CC BY) license (http://creativecommons.org/licenses/by/4.0/). 